\title{
Simulation of $\mathrm{V}(\mathrm{CN})$ Precipitation in Steels Allowing for Local Concentration Fluctuations
}

\author{
Javier Aldazabal $^{1}$, Carlos Garcia-Mateo ${ }^{2}$ and Carlos Capdevila ${ }^{2}$ \\ ${ }^{1}$ CEIT and Tecnun (University of Navarra), Paseo Manuel de Lardizábal 15, 20018 San Sebastián, Spain \\ ${ }^{2}$ MATERALIA Research Group, Department of Physical Metallurgy, Centro Nacional de Investigaciones Metalúrgicas \\ (CENIM-CSIC), Avda. Gregorio del Amo 8, E-28040 Madrid, Spain
}

From a "macroscopic" point of view, steel composition is assumed to vary smoothly along its microstructure. A closer look reveals that, at the atomic level, the material composition does not change so smoothly. Single atoms jump randomly along the crystal lattice due to their thermal energy, therefore creating sporadic zones of the crystal with higher concentration of certain elements. This composition fluctuations are responsible of many phenomena, such as precipitation, Ostwald ripening, some phase transformations. This work proposes a model to simulate the precipitation of $\mathrm{V}(\mathrm{CN})$ precipitates in microalloyed steels in the range of warm temperatures $\left(800-900^{\circ} \mathrm{C}\right)$; when the matrix is fully austenitic (fcc), and taking into account for local composition fluctuations. The model works by dividing the space into very small cells, each containing a single fcc atomic cell. If during the random movement of atoms a small group of adjoining cells reach some critical composition, a nucleus of $\mathrm{V}(\mathrm{CN})$ appears. At the same time, if a cell touching an already existing precipitate reaches some critical vanadium composition, it is very easy to stick it to the precipitate by changing its "phase". But it is also possible that some atoms escape from the precipitate by jumping to the austenitic matrix. The model considers both processes happening simultaneously, and which one is leading depends on the atoms energy, i.e. system temperature, leading to different possible situations, nucleation phenomena, Ostwald ripening or dissolution of precipitates. [doi:10.2320/matertrans.47.2732]

(Received May 22, 2006; Accepted July 14, 2006; Published November 15, 2006)

Keywords: precipitation, carbides, modelling, phase transformation kinetics, diffusion

\section{Introduction}

Nowadays in conventional steel hot-forming processes, microalloying with $\mathrm{V}$-Ti has become a main procedure to obtain ferritic-pearlitic steels with good strength-toughness

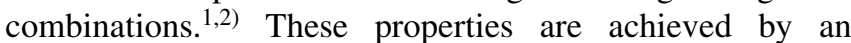
adequate austenite grain size control, by means of fine $\mathrm{Ti}$ precipitates pinning the grain boundaries, and by precipitation strengthening of very fine $\mathrm{V}(\mathrm{CN})$ particles during cooling.

In the case of warm working, the low soaking temperatures applied (usually under $900^{\circ} \mathrm{C}$ ) lead to fine austenitic microstructures and, in consequence, the microaddition of $\mathrm{Ti}$ is not required. On the other hand, since the low reheating temperature does not allow the complete dissolution of $\mathrm{V}(\mathrm{CN})$ precipitates, not all of the vanadium will contribute to the final strength of the steel via precipitation hardening, limiting its possible application as a microalloying element. Nevertheless, if an appropriate $\mathrm{V}(\mathrm{CN})$ precipitation is obtained in the as-rolled product, vanadium can play different roles during warm forging processes, which can lead to a significant improvement in the final mechanical behaviour. Depending on the reheating temperature, which will define the amount of undissolved precipitates, vanadium microalloying can control austenite grain growth during reheating, delay the static recrystallization of austenite and induce precipitation strengthening. ${ }^{3-6)}$

On the other hand, the pinning forces caused by particles and by solutes are responsible for the retardation of recrystallization. ${ }^{7-9)}$ In conventional hot working of microalloyed steels, strain-induced precipitation is the main procedure to obtain the suppression or retardation of recrystallization. In this case, after soaking, the microalloying element is in solution and, during the deformation at lower temperatures, the austenite is supersaturated with respect to the element, promoting its precipitation. In contrast, in the case of warm working of $\mathrm{V}$ microalloyed steels, those particles, which have not been dissolved during a previous reheating treatment, will also act in the steel microstructure, both during deformation and recrystallization, and in combination with the remaining vanadium in solution.

As a first step towards a more general approach to simulate the just described situation, this paper proposes a model to simulate the precipitation of $\mathrm{V}(\mathrm{CN})$ in microalloyed steels in the range of warm temperatures $\left(800-900^{\circ} \mathrm{C}\right)$, beginning with a precipitates-free microstructure where all Vanadium is in solid solution in the austenite.

\section{Model Description}

The starting point for the model consists of a predefined volume, where atoms of different species are randomly distributed. Substitutional atoms in the matrix are able to jump from lattice to lattice sites, and interstitial elements can jump between interstitial positions. These jumps, which take place randomly, are responsible for local sporadic composition fluctuations. When a cell reaches a previously defined critical composition, the model assumes that a $\mathrm{V}(\mathrm{CN})$ nucleus precipitates in that cell. Once a precipitate appears, the probability of $\mathrm{V}$ atoms sticking on its surface increases, leading to the growth of the precipitate. At the same time, the model allows for a very small number of $\mathrm{V}$ atoms to escape from the surface of the precipitate to the austenitic matrix. The model assumes that there is no movement of Vanadium within the precipitates. The following subsections provides with further detail about the model working procedure.

The portion of material studied in each simulation 
Table 1 Compositions used for simulate four different microalloyed steels [atomic fraction].

\begin{tabular}{lcccc}
\hline & Fe & V & C & N \\
\hline High V & $9.56 \times 10^{-1}$ & $1.61 \times 10^{-2}$ & $2.49 \times 10^{-2}$ & $3.03 \times 10^{-3}$ \\
Mid-high V & $9.70 \times 10^{-1}$ & $8.41 \times 10^{-3}$ & $1.95 \times 10^{-2}$ & $1.53 \times 10^{-3}$ \\
Mid-low V & $9.77 \times 10^{-1}$ & $4.53 \times 10^{-3}$ & $1.68 \times 10^{-2}$ & $7.70 \times 10^{-4}$ \\
Low V & $9.81 \times 10^{-1}$ & $2.58 \times 10^{-3}$ & $1.54 \times 10^{-2}$ & $3.90 \times 10^{-4}$ \\
\hline
\end{tabular}

Table 2 Equilibrium composition fixed for the austenitic matrix and for precipitates [atomic fraction].

\begin{tabular}{lcccc}
\hline & $\mathrm{Fe}$ & $\mathrm{V}$ & $\mathrm{C}$ & $\mathrm{N}$ \\
\hline Matrix & $9.85 \times 10^{-1}$ & $6.19 \times 10^{-4}$ & $1.40 \times 10^{-2}$ & $3.90 \times 10^{-6}$ \\
Precipitates & $2.28 \times 10^{-3}$ & $5.19 \times 10^{-1}$ & $3.77 \times 10^{-1}$ & $1.01 \times 10^{-1}$ \\
\hline
\end{tabular}

corresponds to $1 \times 1 \mu \mathrm{m}$ ( $2 \mathrm{D}$ model). The system temperature was fixed to $835^{\circ} \mathrm{C}$, at which austenite lattice parameter is $0.364 \mathrm{~nm}$, and so, the size of each cell is $0.364 \times 0.364 \mathrm{~nm}$. The total number of cells used to discretize the microstructure simulated in this work is $7.52 \times 10^{6}$.

The simulation of $\mathrm{V}(\mathrm{CN})$ precipitation processes was performed in four different microalloyed steels, see Table 1. Steels compositions were selected so under equilibrium conditions the $\mathrm{V}(\mathrm{CN})$ fractions were $0.016,0.008,0.004$ and 0.002 for the high, mid-high, mid-low and low $\mathrm{V}$ microstructures respectively, see Table 2 . For the equilibrium calculations a software for thermodynamics calculations, MTData, was used. ${ }^{10)}$

Proposed model was implemented in a Linux platform using $\mathrm{C}$ programming language. As the code knows exactly the position and state of each Vanadium atom, the memory needed to run different simulations changes with composition, even if the size of the microstructure is the same. Memory requirements for each simulation ranges for $46 \mathrm{MB}$, for the low V microstructures to $51 \mathrm{MB}$ for the high V content simulation. There were necessary $2966 \mathrm{cpu}$ hours to generate all results shown in this work (using Pentium $4 @ 3.0 \mathrm{GHz}$ processors).

Its necessary to highlight the fact that this model is not restricted to the aforementioned conditions, but it is also possible to simulate different situations as bigger volumes, different cell sizes, compositions, temperatures.

\subsection{Initial microstructure}

The first step in order to simulate $\mathrm{V}(\mathrm{CN})$ precipitation is to set the volume size to deal with, in this particular case $s$ $1 \times 1 \mu \mathrm{m}$ was selected. For simplicity, from a computational point of view, results presented in this work corresponds to two-dimensional simulations. Once the volume size to be simulated is determined, it is necessary to discretize it into small elements.

It is known that, for the steels and temperature aimed in this work, the microstructure is composed by austenite (matrix) and $\mathrm{V}(\mathrm{CN}),{ }^{10)}$ both phases with a fcc structure. Thus it is possible to discretize the simulated volume using identical crystallographic cells. Each one corresponding to a fcc unitary cell, with four lattice sites that can only be occupied either by $\mathrm{Fe}$ or $\mathrm{V}$ atoms and four octahedrical interstitial positions that could be filled with either $\mathrm{C}$ and $\mathrm{N}$ atoms.

Once the initial conditions are set, i.e. system composition, and the volume size to be simulated, the total number of lattice sites can be calculated. For conditions used in this paper the microstructure contains $3 \times 10^{7}$ lattice sites.

Fe and $\mathrm{V}$ atoms are randomly dispersed in the microstructure, restricted to the satisfaction of system composition and occupying all lattice sites. Once the substitutional elements are located, the octahedrical sites are filled up with interstitial elements, $\mathrm{C}$ and $\mathrm{N}$, fulfilling the prefixed chemical composition.

\subsection{Atomic movements}

Atoms movement is completely random, and this is accomplished by means of a random-walk scheme. ${ }^{11)}$ These movements lead, in a higher scale, to the diffusion phenomenon.

Statistically it might be the case that an atom tries to escape from the simulated volume through one of its sides, in order to avoid this eventuality, periodical boundary conditions were used during this stage. ${ }^{12)}$ By doing so these atoms are reallocated into the volume through the opposite side.

The movement of substitutional and interstitial atoms were simulated following two different approaches.

For substitutional atoms, there are two options: swapping between atoms (which is highly improbable due to the high energy required), or the movement of vacancies through the lattice. At the studied temperature, $835^{\circ} \mathrm{C}$, austenite vacancies concentration is about $1.92 \times 10^{-6},{ }^{13)}$ in other words, for a simulated microstructure of $1 \times 1 \mu \mathrm{m}$, containing more than 30 million lattice sites, there are only 57 vacancies. From a computational point of view, it is much more effective to simulate the diffusion by swapping substitutional atoms than moving vacancies, although thermodynamically is not. The results obtained using both methods, swapping substitutional atoms and moving vacancies, are indistinguishable. ${ }^{14)}$ By fitting random walk results with empirical measurements ${ }^{15)}$ it is possible to calculate that, each Vanadium atom jumps $3.73 \times 10^{2}$ times per second at $835^{\circ} \mathrm{C}$.

In relation with the movement of interstitial atoms, $\mathrm{C}$ and $\mathrm{N}$, the fraction of interstitial sites filled by both is smaller, and then, the diffusion mechanism is controlled by jumps between octahedrical positions. By matching random walk results with those obtained by solving continuum equation, using Dictra software, ${ }^{16)}$ it is possible to deduce the jump rate for each kind of atom. At the temperature of $835^{\circ} \mathrm{C}$, each $\mathrm{C}$ and $\mathrm{N}$ atom jumps $1.41 \times 10^{8}$ and $4.43 \times 10^{7}$ times per second respectively. ${ }^{14)}$

From these results it is clear that the movement of interstitial species is about 5 orders of magnitude faster than the movement of substitutional atoms. Due to this big difference it is safe for the model to assume that the nucleation and grow or $\mathrm{V}(\mathrm{CN})$ precipitates is limited by the $\mathrm{V}$ diffusion.

\subsection{Nucleation condition}

Those atoms random jumps are responsible for composition fluctuations in each cell. As it was mentioned, the starting point for the simulation is to assume that all $\mathrm{V}$ is in 
solid solution in the matrix (austenite). If during calculations the conditions for a precipitate are fulfilled, i.e. all substitutional sites of a matrix region, equal or bigger than the critical nucleation size, are filled with $\mathrm{V}$ atoms, then the model switches all cells from that region from being matrix to being a $\mathrm{V}(\mathrm{CN})$ precipitate. The critical size was set at $0.41 \mathrm{~nm}$, which corresponds to a single cell in the model. This critical size value is based on empirical TEM measurements in steels with compositions similar to those simulated. ${ }^{17)}$ Different values of the critical size will result on different nucleation behaviours.

\subsection{Precipitates evolution}

To simulate the precipitates evolution the present model makes use of two different variables.

On the one hand, the model assumes that there are three different kind of fcc cells. The first one corresponds to matrix cells, where the main compound is Fe. The second corresponds to precipitates, and its main compound is $\mathrm{V}$, and finally the last type of cell corresponds to interface cells, with the same composition as the matrix, but located arround the precipitates. These interface cells play an important role in the model because they define the zone where $\mathrm{V}$ atoms adhere to, or escape from, $\mathrm{V}(\mathrm{CN})$ precipitates.

On the other hand the second variable that the model handles is related to individual $\mathrm{V}$ atoms. These atoms are able to have one of the two following states. The first one corresponds to free, this state corresponds to $\mathrm{V}$ that is in solid solution in the matrix, and it is able to move freely through the microstructure. The second state, known as fixed, takes into account for those $\mathrm{V}$ atoms within the precipitates, that are not usually allowed to move through the simulated volume.

Figure 1 shows special jumps that proposed model takes into account. First, model assumes that free atoms that jump into an interface cell have certain probability, $R_{\text {stck }}$, of getting stuck to the precipitate, changing its state from free to fixed Fig. 1(a). On the other hand, fixed atoms have a probability, $R_{\text {rel }}$, of getting free, being able to escape from precipitates Fig. 1(b) or from interface Fig. 1(c). Finally, model assumes that all atoms, including fixed ones, are capable of moving along the interface despite its state Fig. 1(d).

It is possible to simulate different phenomena according to the probabilities used for sticking and releasing atoms, and the matrix and precipitate $\mathrm{V}$ concentration. ${ }^{14)}$ Equation 1 shows the relation between these four parameters and the subsequent effect produced.

$$
\begin{array}{ll}
F_{V p r e c} \cdot R_{\text {rel }}>F_{V m t r x} \cdot R_{\text {stck }} & \text { (dissolution) } \\
F_{V p r e c} \cdot R_{\text {rel }} \approx F_{V m t r x} \cdot R_{\text {stck }} & \text { (equilibrium) } \\
F_{V p r e c} \cdot R_{\text {rel }}<F_{V m t r x} \cdot R_{\text {stck }} & \text { (precipitation) }
\end{array}
$$

Where $F_{\text {Vprec }}$ corresponds to the $\mathrm{V}$ equilibrium atomic fraction in the precipitate, $F_{V m t r x}$ to the $\mathrm{V}$ equilibrium atomic fraction in the austenitic matrix, $R_{r e l}$ to the probability for a fixed $\mathrm{V}$ atom of jumping to the interface or to the matrix and finally $R_{\text {stck }}$ to the probability for a free atom to get fixed when jumps to the interface.

In order to calculate the relation between $R_{r e l}$ and $R_{\text {stck }}$ it was necessary to use the equilibrium compositions of the precipitates and the matrix, given by thermodynamics (a)
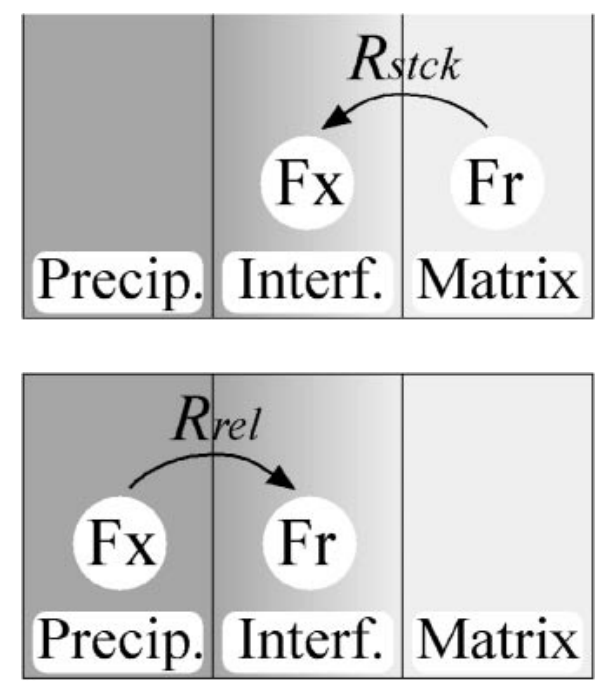

(b)

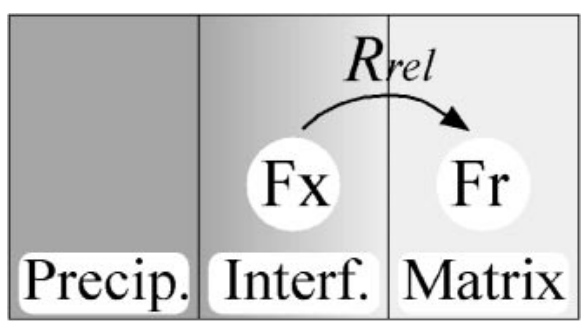

(c)

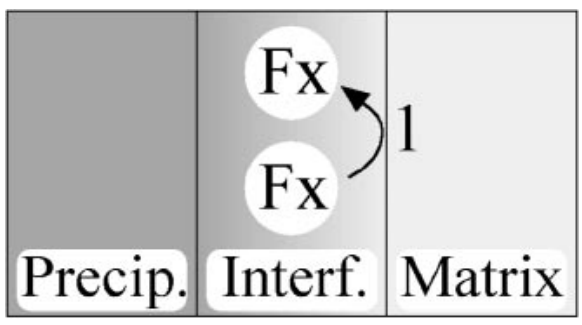

Fig. 1 Atomic jumps considered by the model. Fx and Fr represents fixed and free atoms respectively and the legend close to the arrow indicates the probability of that specific jump.

calculations $^{10)}$ and the equilibrium condition given by eq. (1). At the beginning of the simulation, as microstructure is fully austenitic, the matrix $\mathrm{V}$ concentration is bigger than that expected at equilibrium. Therefore the second terms in previous equations becomes bigger that the first ones, resulting in a precipitation condition. As the system evolves, and precipitates appear, the matrix decreases its $\mathrm{V}$ concentration, reaching the equilibrium when both terms in eq. (1) become equal.

It is very important to highlight that the model reaches a dynamic equilibrium, in the sense that $\mathrm{V}$ atoms are released from, and stuck to, precipitates at identical rates. This phenomenon results in a constant composition and volume fraction of precipitates.

\section{Model Results}

For all the listed steels in Table 1, four different simulations were performed by changing the sequence of pseudo-random numbers used for distributing atoms and for the random walk algorithm. The results shown in this paper 


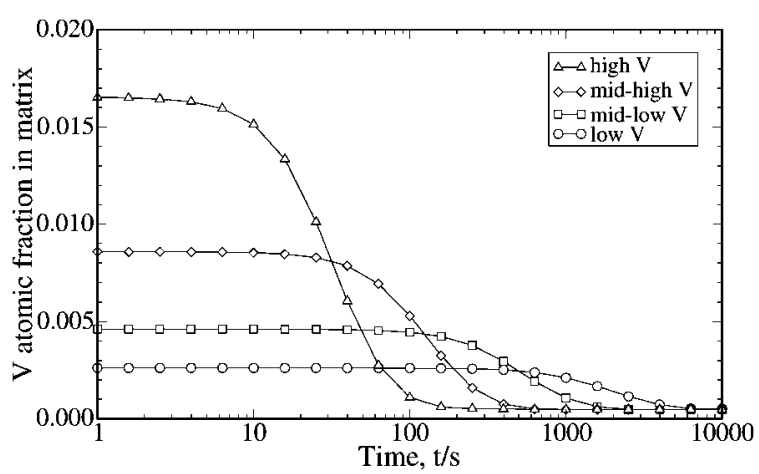

Fig. 2 Predicted V atomic fraction evolution for the four different V steels.

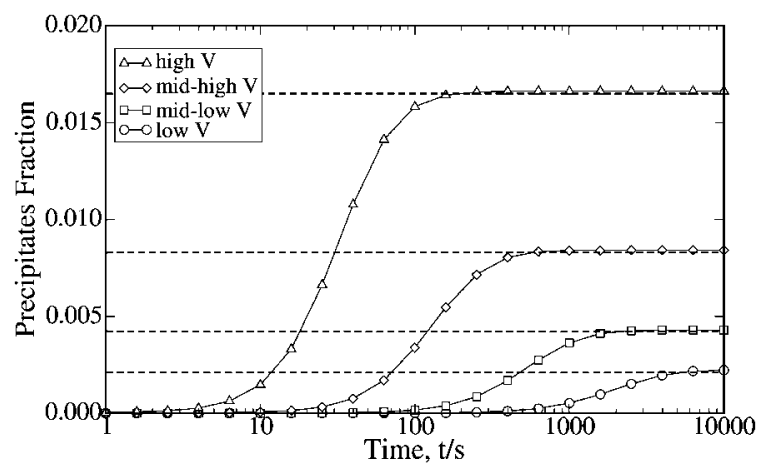

Fig. 3 Predicted precipitate fraction and this expected from thermodynamics calculations.

were obtained by averaging these four simulations for each composition.

\subsection{Simulation parameters}

Once the size of the simulated volume is determined, it is necessary to fix the equilibrium composition of the matrix and of the precipitate. In this case, these concentrations were obtained combining empirical measurements ${ }^{17)}$ and thermodynamic calculations performed using MTData. ${ }^{10)}$ Table 2 shows the equilibrium compositions used for the austenitic matrix and precipitates at $835^{\circ} \mathrm{C}$.

\subsection{Microstructure Evolution}

Figure 2 represents the temporal evolution of the austenite $\mathrm{V}$ concentration for steels listed in Table 1. The model assumes that all fcc cells have the same size and it does not takes into account for the existing differences in lattice parameters between austenite and the precipitates. With this assumption it is possible to compute the precipitates fraction just by counting the number of cells that corresponds to precipitates and knowing the total number of cells of the whole microstructure, Fig. 3. It is also possible to calculate the average size of precipitates just by counting the amount of $\mathrm{V}(\mathrm{CN})$ particles and the total number of precipitates cells, it has to be noted that $\mathrm{V}(\mathrm{CN})$ precipitates might be composed by several cells, depending on its size. Figure 4 shows the evolution of average precipitate sizes for the four compositions.

As the initial microstructure, with no precipitates and all $\mathrm{V}$ in solid solution, evolves, and rich $\mathrm{V}$ precipitates appear,

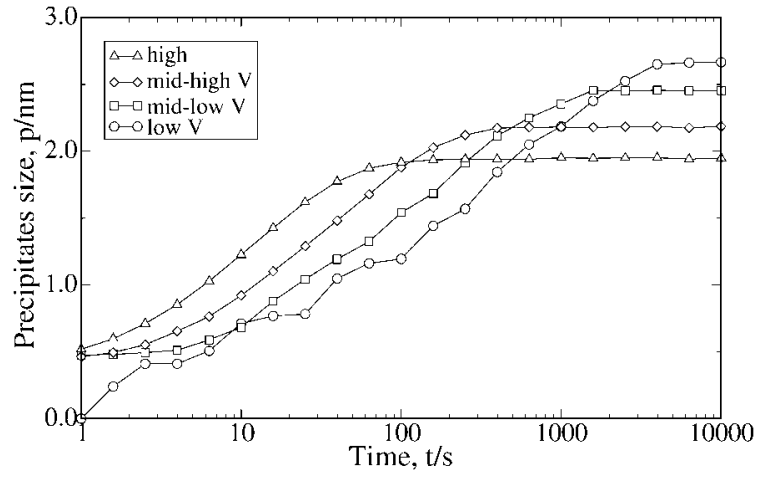

Fig. 4 Evolution of average precipitate sizes along time.

Fig. 2, resulting in a decrease of the Vanadium content of the matrix. At the final stage, when the maximum precipitate fraction is reached, see Fig. 3, the V concentration in the matrix corresponds approximately to the previously fixed equilibrium value, Fig. 2.

An important result that derives from Fig. 3 is that precipitation is not instantaneous, requiring of some incubation time. No parameter was introduced into the model to simulate this time, and it simply appears as the time needed to get enough $\mathrm{V}$ atoms together, moving randomly through the austenitic matrix, for producing a nuclei. It is also evident that as $\mathrm{V}$ content of the microstructure increases it becomes easier to get enough atoms together to generate nuclei, therefore decreasing the incubation time and that needed to obtain the equilibrium fraction, as clearly shown in Fig. 3.

After the incubation time is passed, and the microstructure exhibits some precipitate nuclei, the model allows them to grow until the remaining $\mathrm{V}$ in the matrix satisfies the equilibrium condition given by equation 1 , see Fig. 4 . The high $\mathrm{V}$ content microstructures begin with a size bigger than zero due to the nucleation of small precipitates at very early stagesof the simulation, not represented in the Fig. 4. The only microstructure that begins with an average precipitate size of zero is the one with the lowest initial V concentration. When $\mathrm{V}$ concentration in the steel is high enough, it is easy to generate a very big number of nuclei. A high density of nuclei leads to small average precipitate size at the end of the simulation. In microstructures with a small amount of $\mathrm{V}$ at the beginning, generation of precipitates is more difficult, leading to bigger final sizes. Average precipitates sizes predicted by this model are not far from results obtained by other authors using analytical models, ${ }^{18)}$ at least for times up to $10^{5}$ seconds.

Two are the reasons that accelerate precipitate growth, first an increasing $\mathrm{V}$ concentration on the matrix, and second a higher fraction of precipitates present at the microstructure, atoms have to travel shorter distances to get in contact with a $\mathrm{V}(\mathrm{CN})$ particle. The combination of both effects leads to a very quick increase in precipitate fraction and size for the High V steel, and for the same reasons, the opposite behaviour is found in the Low V steel, see Figs. 3 and 4.

Results predicted in Fig. 3 clearly fits an Avrami type equation of the type.

$$
\xi=1-\exp \left(-c t^{n}\right)
$$


Table 3 Avrami best fitting parameters for simulated microstructures, and time necessary to get a $50 \%$ of the total precipitation.

\begin{tabular}{lcccc}
\hline & $c$ & $n$ & Corr.Coef. & $\mathrm{t}_{0.5}[\mathrm{~s}]$ \\
\hline High V & $5.388 \times 10^{-6}$ & 1.563 & 0.9521 & $3.050 \times 10^{1}$ \\
Mid-High V & $3.914 \times 10^{-5}$ & 1.569 & 0.9521 & $1.205 \times 10^{2}$ \\
Mid-Low V & $3.507 \times 10^{-4}$ & 1.569 & 0.9520 & $4.880 \times 10^{2}$ \\
Low V & $3.071 \times 10^{-3}$ & 1.567 & 0.9520 & $1.796 \times 10^{3}$ \\
\hline
\end{tabular}

where $\xi$ is the normalised precipitate fraction, calculated by dividing the instantaneous fraction by the final one, $c$ and $n$ are constants for a given temperature and composition and $t$ is the time in seconds. In Table 3 are summarised the values that best fit model predictions with the Avrami expression.

The correlation coefficients are bigger than 0.95 for all simulated systems, and the Avrami exponents, $n$, have a value of about 1.56 for all compositions. The literature reports, for diffusion controlled processes, exponents of 1.5 when there is no nucleation and all nuclei appear at the same instant, and values ranging from 1.5 to 2.5 when there is a decreasing nucleation rate. ${ }^{19)}$ Predictions done by the model show a high nucleation rate at very early stages, slowing down when the matrix gets poorer in $\mathrm{V}$, which is in agreement with the reported $n$ values.

\section{Conclusions}

The proposed model is able to simulate properly the evolution of $\mathrm{V}$ content of the matrix during the precipitation, although the model does not contain any specific knowledge about the precipitation mode it is capable to reproduce it as a nucleation and growth process, which is in agreement with the metallurgical understanding.

The precipitate average sizes obtained by the proposed model are in good agreement with those obtained by other authors using analytical models.

\section{Acknowledgments}

Authors would like to thank the Spanish Ministry of
Science and Technology (MCyT) for the financial support. J. Aldazabal thanks for the sponsorship under the project MAT2003-04314 and C. Garcia-Mateo for the financial support in the form of a Ramón y Cajal contract (Programa RyC 2004).

\section{REFERENCES}

1) M. Korchynsky and J. R. Paules: SAE Technical Paper number 890801 (1989).

2) S. Engineer and B. Huchtemann: Fundamentals and Applications of Microalloying Forging Steels, (In: Van Tyne CJ, Krauss G, Matlock DK, editors. TMS, Warrendale, PA, 1996) pp.61-78.

3) C. Garcia-Mateo, J. L. Romero and J. M. Rodriguez-Ibabe: Proceedings of the 41st Mechanical Working and Steel Processing Conference 37 (1999) 653.

4) C. Garcia-Mateo, J. L. Romero and J. M. Rodriguez-Ibabe: Mater. Sci. Forum 435 (1998) 284-286.

5) C. Garcia-Mateo, B. Lopez and J. M. Rodriguez-Ibabe: Scripta Mater. 42 (1999) 137-143.

6) C. Garcia-Mateo, B. Lopez and J. M. Rodriguez-Ibabe: Mater. Sci. Eng. A 303 (2001) 216-225.

7) S. S. Hansen, J. B. Vander Sande and M. Cohen: Metall. Trans. 11A (1980) 387-402.

8) H. L. Andrade, M. G. Akben and J. J. Jonas: Metall. Trans. 14A (1983) 1967-1977.

9) O. Kwon and A.J. DeArdo: Acta Mater 39 (1991) 529-538.

10) MT-DATA: Phase diagram Calculation software (National Physical Laboratory. Teddington, UK, 2003).

11) P. G. Shewmon: Diffusion in solids (McGraw-Hill, 1963) pp. 47-54.

12) D. Raabe: Computational materials science: simulation of materials, microstructures and properties (Wiley-VCH. 1998) pp. 102-104.

13) M. E. Glicksman: Diffusion in solids: field theory, solid-state principles and applications (John Wiley \& Sons Inc. 2000) pp. 228-233.

14) J. Aldazabal and C. Garcia-Mateo: Mater. Sci. Forum. 719 (2005) 500501.

15) J. Gisen and C. Herzig: Z Metallk. 78 (1987) 291-294.

16) Dictra User's Guide (Dept. Of Materials Science and Engineering, Royal Institute of technology, Sweden, 1998) pp. 63-71.

17) C. Garcia-Mateo: Influencia de la aleación con $V$ en el conformado en tibio de aceros de contenido medio/alto en $C(\mathrm{PhD}$ Thesis, University of Navarra, San Sebastián. 2000) pp. 4.46-4.49.

18) P. Maugis and M. Gouné: Acta Mater 53 (2005) 3359-3367.

19) J. W. Christian: The theory of transformations in metals and alloys (Pergamon Press, 1975) pp. 542. 